\title{
Strong KDM4B and KDM4D Expression Associates with Radioresistance and Aggressive Phenotype in Classical Hodgkin Lymphoma
}

\author{
HAMID BUR ${ }^{1}$, KIRSI-MARIA HAAPASAARI ${ }^{2}$, TAINA TURPEENNIEMI-HUJANEN ${ }^{1}$, \\ OUTI KUITTINEN $^{1}$, PÄIVI AUVINEN ${ }^{3}$, KATJA MARIN ${ }^{3}$, YLERMI SOINI ${ }^{4}$ and PEETER KARIHTALA ${ }^{1}$ \\ Departments of ${ }^{1}$ Oncology and Radiotherapy and ${ }^{2}$ Pathology, Medical Research Center Oulu, \\ Oulu University Hospital and University of Oulu, Oulu, Finland; \\ ${ }^{3}$ Department of Oncology, Cancer Center of Eastern Finland, Kuopio University Hospital, Kuopio, Finland; \\ ${ }^{4}$ Department of Pathology and Forensic Medicine, University of Eastern Finland, \\ Cancer Center of Eastern Finland, Kuopio, Finland
}

\begin{abstract}
Background: Epigenetic regulators, including Jumonji domain 2 (JMJD2/KDM4) proteins are involved in post-translational modification of histone demethylation and have a major role in carcinogenesis of many solid tumors. Materials and Methods: We assessed immunohistochemically the expression of lysine $(K)$-specific demethylase 4 (KDM4)A, KDM4B and KDM4D in tumors from 91 patients of adriamycin, bleomycin, vinblastine, darcabazine (ABVD)treated classical Hodgkin lymphoma. Results: Strong cytoplasmic KDM4B expression in the reactive cellular infiltrate and also in Reed-Sternberg (RS) cells predicted poor relapse-free survival (RFS) $(p=0.020$ and $p=0.022$, respectively) in patients with limited-stage disease. Strong $K D M 4 B$ expression in $R S$ cells was also related to $B$ symptoms $(p=0.007)$ and advanced stage $(p=0.024)$. Strong KDM4D expression in the cytoplasm of RS cells was also associated with poor RFS in limited-stage patients RFS $(p=0.043)$ and, most significantly, in patients receiving involved-field radiotherapy ( $p=0.007$ ). Conclusion: $K D M 4 B$ and KDM4D expression may associate with an aggressive subtype of classical Hodgkin lymphoma and be linked with radioresistance.
\end{abstract}

This article is freely accessible online.

Correspondence to: Peeter Karihtala, Department of Oncology and Radiotherapy, Oulu University Hospital, PO Box 22, 90029 Oulu, Finland. Telephone: +358 83152011, Fax: +358 83156449, e-mail: peeter.karihtala@oulu.fi

Key Words: Hodgkin lymphoma, KDM4B, KDM4D, radioresistance.
The prognosis of Hodgkin lymphoma patients has improved markedly during the last decades. However, $20 \%$ of patients with classical Hodgkin lymphoma still suffer from relapse. Therefore, it is utmost important to find more information over the biological characteristics of the disease in different individuals to accurately identify the patients for whom more effective first-line treatments are indicated (1).

Epigenetic alterations are independent of changes in the primary DNA sequence but still able to modify the expression of various genes. Epigenetic modifications include the methylation of cytosine bases in DNA, changes in miRNA expression, changes in the positioning of nucleosomes along the DNA and post-translational modifications of histone proteins, which are in the focus of the current study $(2,3)$.

Negatively charged DNA twists around a core of positively charged histones allowing condensation of the genetic material. The state of DNA condensation varies according to the specific alterations in post-translational histone modifications. Acetylation and methylation are the most predominant histone protein regulators. In acetylation, the positively charged lysine lowers the charge of a histone leading to the relaxation of chromatin, which improves gene transcription. In methylation, the methyl groups are transferred to arginine or lysine residues and, while the charge of the histone is not altered, the gene expression is activated $(4,5)$.

Histone methylation is reversible (6). There are two main enzyme families that can demethylate histones. Lysinespecific demethylases (LSD1 and 2) were the first known histone demethylases (7). The other family is Jumonji C (JMJC) histone demethylating enzymes in which 18 of 30 members have histone demethylase activity. All these proteins have the JMJC domain but, depending on the degree 
Table I. Immunohistochemical methods.

\begin{tabular}{|c|c|c|c|}
\hline Primary antibody & Source of primary antibody & Dilution & Immunostaining method \\
\hline $\begin{array}{l}\text { KDM4A } \\
(\text { ab104831) }\end{array}$ & $\begin{array}{l}\text { Abcam plc, } \\
\text { Cambridge, UK }\end{array}$ & $1: 2,000$ & $\begin{array}{l}\text { Dako REAL }^{\mathrm{TM}} \text { EnVision }{ }^{\mathrm{TM}} \text { Detection System } \\
\text { (Dako Denmark A/S, Glostrup, Denmark) }\end{array}$ \\
\hline $\begin{array}{l}\text { KDM4B } \\
(\text { ab103129) }\end{array}$ & $\begin{array}{c}\text { Abcam plc, } \\
\text { Cambridge, UK }\end{array}$ & $1: 225$ & $\begin{array}{l}\text { Dako REAL }^{\mathrm{TM}} \text { EnVision }{ }^{\mathrm{TM}} \text { Detection System } \\
\text { (Dako Denmark A/S, Glostrup, Denmark) }\end{array}$ \\
\hline KDM4D (ab93694) & $\begin{array}{c}\text { Abcam plc, } \\
\text { Cambridge, UK }\end{array}$ & $1: 1,500$ & $\begin{array}{l}\text { Dako REAL }^{\mathrm{TM}} \text { EnVision }{ }^{\mathrm{TM}} \text { Detection System } \\
\text { (Dako Denmark A/S, Glostrup, Denmark) }\end{array}$ \\
\hline
\end{tabular}

KDM4, Lysine (K)-specific demethylase.

of homology and the presence of other domains, the members are also classified into subfamilies (8). One biologically highly important subfamily is lysine $(\mathrm{K})$-specific demethylase 4 (KDM4; JMJD2) having the roughly 150aminoacid-long JMJC domain, which, in turn, interacts with JMJN domain creating the catalytic component that demethylates histones by way of the dioxygenase reaction (9). KDM4 family proteins can demethylate tri-, di- and monomethylated lysine residues, which have a number of different effects on cell function (7).

This study was undertaken to characterize the previously unexplored roles of KDM4A, KDM4B and KDM4D from previously untreated classical Hodgkin lymphomas. Special emphasis was given to the localization of the expression, association with previously recognized classical prognostic factors and possible usability as a predictive factor.

\section{Materials and Methods}

Patient collection. The material consisted of pre-treatment samples from 91 patients with histologically confirmed classical Hodgkin lymphoma diagnosed between the years 1999-2012. All patients were treated with adriamycin, bleomycin, vinblastine, darcabazine (ABVD) chemotherapy in the first-line setting. After chemotherapy, $68.1 \%$ of the patients received involved-field radiotherapy (IFRT) up to the total dose of 20-40 Gy. Forty-two patients were diagnosed and treated at Oulu University Hospital, 42 at Kuopio University Hospital and seven at the Central Hospitals of Kajaani, Kemi, Kokkola or Rovaniemi. Diagnoses were reviewed by a specialist hematopathologist and the Ethics Committee of the Northern Ostrobothnia Hospital District approved the study design (reference number 42/2010).

Immunohistochemistry. Lymphoma samples were fixed in formalin and embedded in paraffin. Three- $\mu \mathrm{m}$ sections of a representative tumor area from the paraffin blocks were cut and placed on SuperFrostPlus glass slides (Menzel-Gläser, Braunschweig, Germany). The slides were incubated at $+37^{\circ} \mathrm{C}$ for $4 \mathrm{~h}$ before deparaffinization in a clearing agent Histo-Clear (National Diagnostics, Atlanta, GA, USA) and rehydration in a graded series of alcohol solutions. Next, the slides were microwaved for $10 \mathrm{~min}$ in Tris-EDTA solution, $\mathrm{pH} \mathrm{9,} \mathrm{and,} \mathrm{after} 20 \mathrm{~min}$ cooling at room temperature, they were incubated in a $3 \% \mathrm{H}_{2} \mathrm{O}_{2}$ solution for $5 \mathrm{~min}$ to block the endogenous peroxidase activity. Next, the slides were incubated with primary antibody (Table I) in a humidity chamber at room temperature for 1 hour (KDM4A) or over night at $+4^{\circ} \mathrm{C}$ (KDM4B and KDM4D). Immunostaining was continued using a Dako REAL $^{\text {тм }}$ EnVision $^{\text {Tм }}$ Detection System (Dako Denmark A/S, Glostrup, Denmark) according to the instructions of manufacturer. Diaminobenzidine was used to detect the immunoreaction. Between all stages of the immunostaining procedure, the slides were washed with Tris-buffered saline (TBS). Finally, the slides were counterstained with Mayer's hematoxylin (Reagena, Toivola, Finland), dehydrated and mounted with Histomount (National Diagnostics).

Evaluation of immunohistochemical staining. Evaluation of immunostaining was performed by two observers, an experienced hematopathologist (K-M.H.) and (H.B). During the evaluation of the immunostaining, the clinical data was blinded. Immunostaining was graded (i) separately in Reed-Sternberg (RS) cells and in the surrounding reactive cellular infiltrate; (ii) separately in nuclei and cytoplasm; and (iii) separately according to the extent (0-100\%) and the intensity of immunostaining: ( 0 , no immunostaining; 1 , weak immunostaining intensity; 2 , moderate immunostaining intensity; 3 , strong immunostaining intensity; 4, very strong immunostaining intensity).

Statistical analysis. For statistical analyses, the intensity was multiplied with degree of extent of immunostaining (0-100\%) resulting in a continuous variable $0-400$ that was further divided into two classes (low expression and high expression) based on the median expression of each variable. This two-classed variable was used in all statistical analyses.

Two-sided Fisher's exact test was used to determine the association between clinicopathological parameters and protein expression. Associations between epigenetic markers and patient survival were analyzed by using the Kaplan-Meier method; the statistical significance of differences was evaluated by using the logrank test. Relapse-free survival (RFS) was calculated from the date of diagnosis to the date of confirmed relapse. Results were considered as significant if the $p$-value was $<0.05$. The statistical power was not sufficient enough to analyze the overall survival due to the low number of deaths.

\section{Results}

Clinical and histological data are presented in Table II. The median follow-up time was 54 months (range=4-127). After chemotherapy, $61(67 \%), 27(29.7 \%)$ and $3(3.3 \%)$ of the 


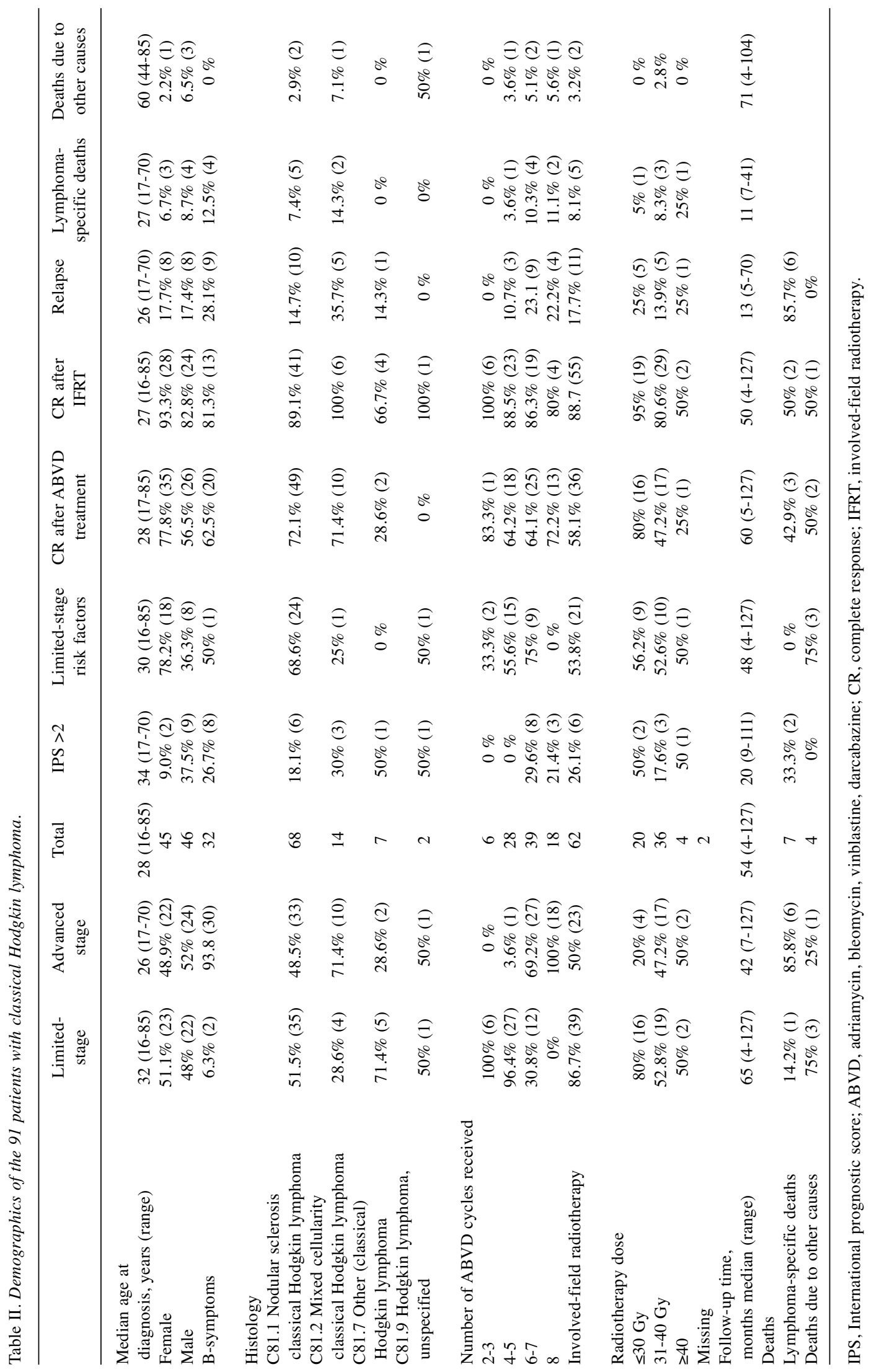



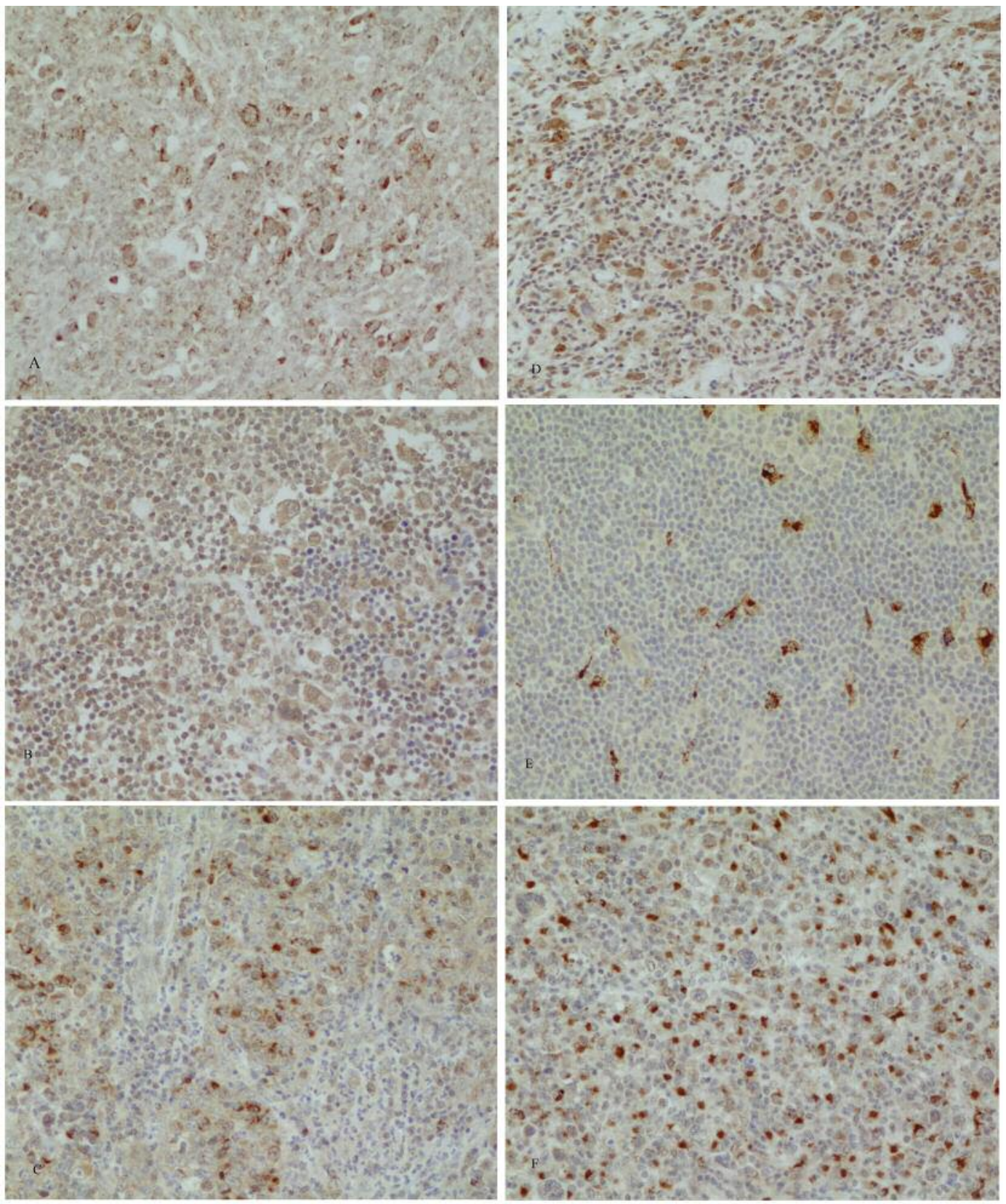

Figure 1. Immunostaining of KDM4B and KDM4D expression in Hodgkin's lymphoma. A. Cytoplasmic KDM4B expression was strong in ReedSternberg (RS) cells. Staining was partly granular. The cytoplasm of reactive cellular infiltrate was also partly positively immunostained. B. Strong $K D M 4 B$ immunostaining in the cytoplasm of $R S$ cells. In reactive cellular infiltrate, immunostaining was moderate throughout. $C$. Very strong $K D M 4 B$ immunostaining in the cytoplasm of RS cells. Staining was partly granular. In reactive cellular infiltrate, immunostaining was mostly negative. D. Strong nuclear KDM4D immunostaining in RS cells. Moderate nuclear immunostaining in some of the reactive cellular infiltrate. E. $K D M 4 D$ immunostaining was very strong and granular in the cytoplasm of most RS cells. Negative staining in reactive cellular infiltrate. F. Very strong KDM4D nuclear immunostaining in reactive cellular infiltrate. All figures are at $\times 20$ magnification.

patients had complete response, partial response and progressive disease, respectively. After radiotherapy, complete response was achieved by $52(83.9 \%)$ of the patients, while 6 (9.7\%) had partial response and one patient had progressive disease. There were 7 (7.7\%) lymphomaspecific deaths and $4(4.4 \%)$ deaths due to other causes (e.g., infections). Sixteen (17.6\%) patients suffered a relapse during the follow-up. 

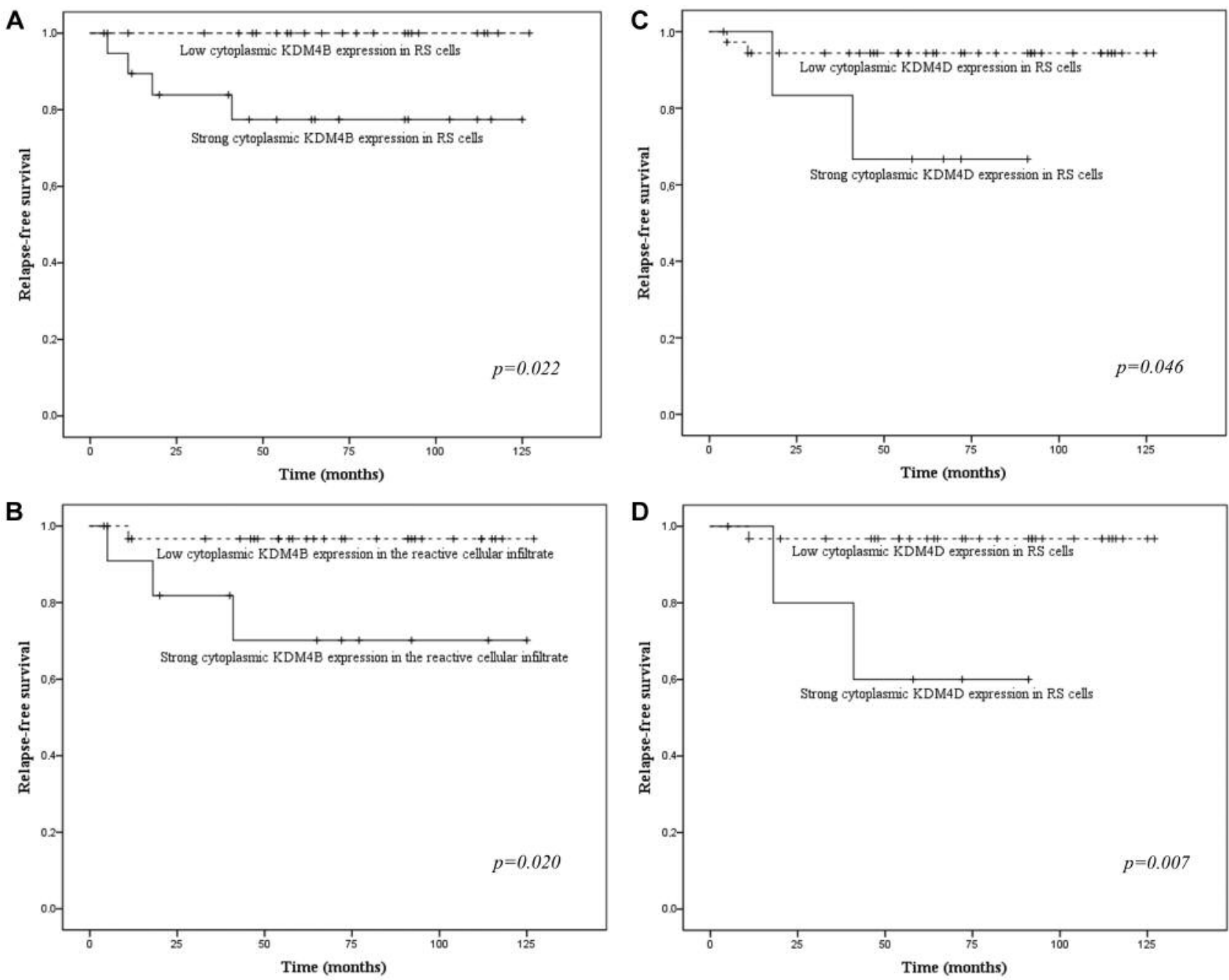

Figure 2. Kaplan-Meier curves showing relapse-free survival in relation to cytoplasmic KDM4B immunostaining in Reed-Sternberg (RS) cells in limited-stage disease (A), cytoplasmic KDM4B immunostaining in reactive cellular infiltrate in limited-stage disease (B), cytoplasmic KDM4D immunostaining in RS cells in limited-stage disease $(C)$ and cytoplasmic KDM4D immunostaining in RS cells after radiotherapy in limited-stage disease $(D)$.

Almost all RS cells (92.2\%) and reactive cellular infiltrates (96.7\%) showed a cytoplasmic KDM4A positivity, with intensity varying from weak to moderate. Nuclear KDM4A expression was detected in the reactive cellular infiltrate in most cases $(83.3 \%)$ and in one case localized only in RS cells.

KDM4B expression was found in the cytoplasm, with intensity varying from weak to strong (Figure 1A-C). KDM4B immunostaining appeared usually as granular. In almost all cases, both RS cells $(93.4 \%)$ and reactive cellular infiltrates $(93.4 \%)$ were positive for KDM4B. The intensity in reactive cellular infiltrate was not associated with the intensity of KDM4B in RS cells. KDM4B also appeared in a few nuclei of RS cells (3.3\% of cases) and reactive cellular infiltrate $(20.9 \%$ of cases).
KDM4D expression was found in the cytoplasm (68.1\%) and nuclei $(25.3 \%)$ of RS cells. In reactive cellular infiltrate, KDM4D expression was found in nuclei (26.4\%) and cytoplasm $(93.4 \%)$. Its intensity varied from weak to very strong in the cytoplasm of RS cells and in reactive cellular infiltrate. Reactive cellular infiltrate staining was usually observed next to RS cells. In most cases, KDM4D staining was granular (Figure 1D-F).

Low nuclear KDM4A expression in RS cells associated with the presence of B-symptoms $(p=0.006)$. Strong cytoplasmic KDM4A expression in reactive cellular infiltrate associated nearly significantly with poorer RFS ( $p=0.054 ; 5-$ year survival in the patients with low expression $92.1 \%$ and in those with high expression 94.1\%). 
Strong cytoplasmic KDM4B expression in multinucleated RS cells was related to the presence of B-symptoms $(p=0.007)$, advanced stage $(p=0.024)$ and higher International Prognostic Score (IPS) scores $(p=0.001)$ in advanced disease. It also predicted poorer RFS in patients with limited-stage disease $(p=0.022)$ (Figure 2A). All patients with low cytoplasmic KDM4B expression were free of relapse during the follow-up, while RFS for the patients with strong KDM4B expression was 79\% at five years. Similarly, the strong cytoplasmic KDM4B expression in the reactive cellular infiltrate predicted worse RFS in patients with limited-stage disease $(p=0.020)$ (Figure 2B); RFS after five years follow-up was $97 \%$ and $75 \%$ in low $v$ s. strong KDM4B expression, respectively.

Strong KDM4D expression in the cytoplasm of RS cells was associated with dismal RFS among the limited-stage patients (five-year RFS 71\% vs. 94\%; $p=0.046$ ) (Figure 2C). Strong cytoplasmic KDM4D expression also predicted worse RFS in limited-stage patients who had received IFRT (fiveyear RFS 60\% vs. 97\%; $p=0.007$ ) (Figure 2D). On the other hand, in those limited-stage patients who had not received IFRT, KDM4D expression did not predict outcome. In addition, KDM4D expression in the cytoplasm of RS cells associated with advanced stage $(p=0.043)$. Stronger nuclear KDM4D expression in reactive cellular infiltrate was associated with the presence of B-symptoms $(p=0.002)$ and advanced disease $(p=0.007)$. As a result of excellent prognosis and a limited number of relapses in this material, reliable multivariate analysis could not be performed.

\section{Discussion}

This is the first study to show that epigenetic posttranslational modifications may have significant prognostic and, possibly, even predictive value in Hodgkin lymphomas. Our observations were noticed only in limited-stage patients where more accurate prognostic markers are particularly needed for the selection of optimal first-line treatment. Epigenetic regulation seems important in RS cells regarding patient survival; however, it may also have an effect on lymphomagenesis in the reactive cellular infiltrate of Hodgkin lymphoma.

There is no previous literature on KDM4 expression in lymphomas. However, KDM4 isoenzymes are overexpressed in breast, colorectal, lung and prostate cancers compared with the corresponding benign tissues and also required for efficient cancer cell proliferation in many solid tumors (10). Existing data also suggest that histone demethylase KDM6B is overexpressed in RS cells compared with surrounding tissues and may also contribute to the pathogenesis of Hodgkin lymphoma (11).

Limited-stage Hodgkin lymphoma patients are treated with short chemotherapy courses that are assumed to cure the subclinical disease outside the radiotherapy field. After chemotherapy, the disease in involved areas is treated with radiotherapy in the patients with limited-stage. The effect of radiotherapy is mainly mediated through the generation of free radicals, which are able to break DNA double-strands, leading ultimately to apoptosis. KDM4D seems to have a significant role in promoting the repair of double-strand breaks, thereby maintaining genome integrity (12). Radiation doses used are relatively low in Hodgkin lymphoma, because the disease is usually highly radiosensitive. Sublethal radiotherapy doses may lead to KDM4D promoting repair of the double-strands and enhance radioresistance. In the present work, the strong KDM4D expression in RS cell cytoplasm associated with poorer RFS in the limited-stage patients but only in those who had received IFRT. All of these patients had their relapse in the radiotherapy field. Although we are unable to definitely confirm this with the current methods, KDM4D overexpression seems to lead to a higher risk for resistance to radiotherapy in Hodgkin lymphomas. There is no previous literature assessing KDM4D as a prognostic factor in lymphomas or in solid tumors, although a recent study showed that in non-small cell carcinoma high KDM4D expression associates with lymph node involvement (13).

KDM4D differs from the other KDM4 family members in its structure and demethylating function (14). Interestingly, KDM4D-based enhanced DNA repair is PARP1-dependent. Since there are already PARP inhibitors in the clinical use of solid malignancies (15), it might offer an interesting possibility to control the development of radioresistance. In favor of this, a recent study also demonstrated that inhibiting PARP1 enhances radiotherapy responses in nasopharyngeal carcinoma (16). If similar results could be found in lymphomas and the results presented here are confirmed in independent studies, the role of PARP inhibitors could be tested in the KDM4D-overexpressing subpopulations of Hodgkin lymphoma.

Oxygen tension is much lower in lymph nodes (0.5-4.5\%) compared to absolute oxygen tension in the bone marrow (12\%) or bloodstream (20\%) (17). This frequently leads to hypoxic conditions in lymph nodes and, interestingly, hypoxia-inducible factor $1 \alpha$ (HIF1) induces KDM4B protein expression (18). Hypoxia is known to associate also with increased failure risk in radiotherapy, especially in low-dose treatments. Therefore, it could be speculated that KDM4B may help RS cells and reactive cellular infiltrate to survive and thrive in a hypoxic environment. This would be in line with our results that demonstrate poorer RFS in the limitedstage patients with strong cytoplasmic KDM4B expression.

To the best of our knowledge, this is the first study to report the prognostic role of KDM4B in any malignancy. KDM4B overexpression has been linked to gastric, bladder, lung and colorectal cancers and is required for proliferation, colony-formation ability, invasion and survival in respective 
cell lines $(19,20)$. The current results show that KDM4B is associated with the traditional prognostic factors of Hodgkin lymphoma, namely B-symptoms and IPS score in advanced stage disease. Since KDM4B plays an important part in the repair of DNA double-strand breaks (DSBs) and also initiates a DNA damage response, it may have an impact on treatment resistance with regards to radiotherapy and certain chemotherapeutic agents (21).

We conclude that low immunohistochemical KDM4B and KDM4D expression may identify the subpopulation of Hodgkin lymphoma patients with limited-stage disease who have an excellent prognosis after standard cytotoxic ABVD therapy and radiotherapy. This may imply that especially KDM4D could be a potential marker of radioresistance in Hodgkin lymphoma. If these findings are confirmed in an independent patient population, the results may lead to clinical studies seeking different variations in treatment protocols or new treatments in limited-stage Hodgkin lymphomas.

\section{Conflicts of Interest}

None.

\section{Funding}

The Authors thank Oulu University Scholarship Foundation, the Finnish Cancer Society and the Finnish Antituberculosis Association for support.

\section{Acknowledgements}

The Authors thank Anne Bisi for her help with immunohistochemical staining.

\section{References}

1 Stathis A and Younes A: The new therapeutical scenario of Hodgkin lymphoma. Ann Oncol 10: 2026-2033, 2015.

2 Jones PA and Baylin SB: The fundamental role of epigenetic events in cancer. Nat Rev Genet 3: 415-428, 2002.

3 Egger G, Liang G, Aparicio A and Jones PA: Epigenetics in human disease and prospects for epigenetic therapy. Nature 429: 429-457, 2004.

4 Chi P, Allis CD and Wang GG: Covalent histone modifications, miswritten, misinterpreted and miserased in human cancers. Nat Rev Cancer 10: 457-469, 2010.

5 Suganuma $\mathrm{T}$ and Workman JL: Signals and combinatorial functions of histone modifications. Annu Rev Biochem 80: 473499, 2011

6 Huang J, Sengupta R, Espejo AB, Lee MG, Dorsey JA, Richter M, Opravil S, Shiekhattar R, Bedford MT, Jenuwein T and Berger SL: p53 is regulated by the lysine demethylase LSD1. Nature 449: 105-108, 2007.

7 Black JC, Van Rechem C and Whetstine JR: Histone lysine methylation dynamics: establishment, regulation, and biological impact. Mol Cell 48: 491-507, 2012.
8 Kooistra SM and Helin K: Molecular mechanisms and potential functions of histone demethylases. Nat Rev Mol Cell Biol 13: 297-311, 2012.

9 Chen Z, Zang J, Whetstine J, Hong X, Davrazou F, Kutateladze TG, Simpson M, Mao Q, Pan CH, Dai S, Hagman J, Hansen K, Shi Y and Zhang G: Structural insights into histone demethylation by JMJD2 family members. Cell 125: 691-702 2006.

10 Berry WL and Janknecht R: KDM4/JMJD2 Histone Demethylases: Epigenetic Regulators in Cancer Cells. Cancer Res 73: 2936-2942, 2013.

11 Anderton JA, Bose S, Vockerodt M, Vrzalikova K, Wei W, Kuo M, Helin K, Christensen J, Rowe M, Murray PG and Woodman $\mathrm{CB}$ : The H3K27me3 demethylase, KDM6B, is induced by Epstein-Barr virus and over-expressed in Hodgkin's Lymphoma. Oncogene 30: 2037-2043, 2011.

12 Khoury-Haddad H, Guttmann-Raviv N, Ipenberg I, Huggins D, Jeyasekharan AD and Ayoub N: PARP1-dependent recruitment of KDM4D histone demethylase to DNA damage sites promotes double-strand break repair. Proc Natl Acad Sci USA 111: 728$737,2014$.

13 Soini Y, Kosma VM and Pirinen R: KDM4A, KDM4B and KDM4C in non-small cell lung cancer. Int J Clin Exp Pathol: 10: 12922-12928, 2015.

14 Trojer P, Zhang J, Yonezawa M, Schmidt A, Zheng H, Jenuwein $\mathrm{T}$ and Reinberg D: Dynamic Histone H1 Isotype 4 Methylation and Demethylation by Histone Lysine Methyltransferase G9a/KMT1C and the Jumonji Domain-containing JMJD2/ KDM4 Proteins. J Biol Chem 284: 8395-8405, 2009.

15 Reinbolt RE and Hays JL: The Role of PARP Inhibitors in the Treatment of Gynecologic Malignancies. Front Oncol 3: 237, 2013.

16 Chow JP, Man WY, Mao M, Chen H, Cheung F, Nicholls J, Tsao SW, Li Lung M and Poon RY: PARP1 is overexpressed in nasopharyngeal carcinoma and its inhibition enhances radiotherapy. Mol Cancer Ther 12: 2517-2528, 2013.

17 Bertolotti M, Sitia R and Rubartelli A: On the redox control of B lymphocyte differentiation and function. Antioxid Redox Signal 16: 161139-161149, 2012.

18 Beyer S, Kristensen MM, Jensen KS, Johansen JV and Staller P: The histone demethylases JMJD1A and JMJD2B are transcriptional targets of hypoxia-inducible factor HIF. J Biol Chem 283: 36542-36552, 2008.

19 Li W, Zhao L, Zang W, Liu Z, Chen L, Liu T, Xu D and Jia J: Histone demethylase JMJD2B is required for tumor cell proliferation and survival and is overexpressed in gastric cancer. Biochem Biophys Res Commun 416: 372-378, 2011.

20 Toyokawa G, Cho HS and Iwai Y: The histone demethylase JMJD2B plays an essential role in human carcinogenesis through positive regulation of cyclin-dependent kinase 6. Cancer Prev Res 4: 2051-2061, 2011.

21 Mallette FA, Mattiroli F, Cui G, Young LC, Hendzel MJ, Mer G, Sixma TK and Richard S: RNF8- and RNF168-dependent degradation of KDM4A/JMJD2A triggers 53BP1 recruitment to DNA damage sites. The EMBO Journal 31: 1865-1878, 2012.

Received July 15, 2016

Revised July 24, 2016

Accepted August 2, 2016 